\title{
Féeries
}

Études sur le conte merveilleux, XVII $-\mathrm{XIX}{ }^{\mathrm{e}}$ siècle

16 | 2020

Le conte, les mythes antiques, la Bible

\section{Vénus, la Barbe bleue et la Vierge Marie. Enquête intertextuelle et interculturelle sur une étrange filiation (Apulée, Basile, Perrault, La Force, Gretchen Wild et les frères Grimm)}

Venus, Bluebeard and the Virgin Mary. Intertextual and intercultural

investigation on a strange affiliation (Apuleius, Basile, Perrault, La Force, Gretchen Wild and the Grimm Brothers)

\section{Ute Heidmann}

\section{OpenEdition}

Journals

Édition électronique

URL : https://journals.openedition.org/feeries/2682

DOI : $10.4000 /$ feeries. 2682

ISSN : 1957-7753

Éditeur

UGA Éditions/Université Grenoble Alpes

Édition imprimée

ISBN : 978-2-37747-194-2

ISSN : 1766-2842

Référence électronique

Ute Heidmann, « Vénus, la Barbe bleue et la Vierge Marie. Enquête intertextuelle et interculturelle sur une étrange filiation (Apulée, Basile, Perrault, La Force, Gretchen Wild et les frères Grimm) », Féeries [En ligne], 16 | 2020, mis en ligne le 26 novembre 2020, consulté le 10 décembre 2021. URL : http:// journals.openedition.org/feeries/2682; DOI : https://doi.org/10.4000/feeries.2682

Ce document a été généré automatiquement le 10 décembre 2021.

(c) Féeries 


\title{
Vénus, la Barbe bleue et la Vierge
} Marie. Enquête intertextuelle et interculturelle sur une étrange filiation (Apulée, Basile, Perrault, La Force, Gretchen Wild et les frères

\section{Grimm)}

\author{
Venus, Bluebeard and the Virgin Mary. Intertextual and intercultural \\ investigation on a strange affiliation (Apuleius, Basile, Perrault, La Force, \\ Gretchen Wild and the Grimm Brothers)
}

\section{Ute Heidmann}

Cette étude s'attache à éclairer la surprenante parenté qui lie, dans nombre de contes, des histoires et figures provenant de la Bible et des mythes gréco-romains, ici celles de Vénus, de Barbe bleue et de la Vierge Marie. Si cet assemblage, dans lequel se glissent encore une ogresse et une méchante Reine des Fées, paraît presque surréaliste, en raison de son hétérogénéité, l'analyse comparative des contes qui les représentent révèle que leur parenté résulte d'un dialogue intertextuel et interculturel très cohérent et significatif ${ }^{1}$.

\section{De Vénus à l'ogresse napolitaine et à la Barbe bleue}

Dans le texte latin qui raconte les amours de Psyché et Cupidon, la déesse Vénus est peinte sous les traits d'une méchante mégère, traits qui ont durablement marqué nombre de descriptions de belles-mères et de marâtres dans les contes européens. L'ouvrage d'Apulée, plus connu sous le titre L'Âne d'or, se compose de récits variés (uarias fabulas) racontés par un jeune homme transformé en âne par un acte de magie 
maladroit de sa maitresse. Ce narrateur fictionnel prétend avoir entendu raconter, sous sa peau d'âne, la "si jolie petite fable » (tam bellam fabellam) de Psyché par la vieille servante d'une bande de brigands. Érigée en parangon du conte ancien à la Renaissance et à l'Âge classique et omniprésente dans la littérature et l'iconographie des grandes et petites cours de toute l'Europe ${ }^{3}$, la fabella raconte l'histoire d'une jeune princesse si belle que les humains la vénèrent comme une divinité. Vénus, déesse de la beauté en titre, s'en prend par jalousie à la jeune mortelle. Elle ordonne à son fils Cupidon de frapper Psyché d'une flèche qui doit la rendre amoureuse du plus vil des hommes. Elle en dresse une sorte de portrait-robot qui n'échappera pas à l'attention de Perrault, qui l'utilisera pour fabriquer son hideux personnage à la barbe bleue.

3 La narration des aventures et mésaventures de la célèbre Psyché, représentées et interprétées d'innombrables fois, se prête en effet à merveille à l'expérimentation poétique. L'histoire de la jeune femme persécutée par la puissante Vénus, jalouse de la grande beauté de la mortelle, la relation mouvementé avec Cupidon, chargé par sa mère Vénus de punir Psyché, mais qui tombe lui-même amoureux d'elle et reste invisible de peur que sa mère ne les découvre, l'envie de Psyché de le voir et la série d'épreuves de plus en plus dangereuses que la méchante belle-mère inflige à la jeune femme pour la faire mourir, fournissent à Basile, La Fontaine, Perrault, La Force et aux frères Grimm des possibles narratifs infinis pour créer de nouvelles histoires et de nouvelles formes génériques.

4 L'épreuve la plus perfide que la déesse Vénus inflige à la jeune mortelle consiste à l'envoyer aux Enfers avec la mission de chercher « un petit peu de beauté » auprès de Proserpine, grande déesse des morts. Les paroles prêtées à Vénus par le narrateur fictif sous sa peau d'âne indiquent bien la dimension parodique de l'ouvrage d'Apulée à l'égard des dieux romains :

Mais tu me dois encore un service, ma poupette. Prends cette capsule (elle la lui donna), et va-t'en de suite aux Enfers, jusqu'aux pénates enténébrées d'Orcus. Tu tendras la capsule à Proserpine et tu lui diras « Vénus te fait dire de lui envoyer un petit peu de ta beauté, au moins la dose pour une journée seulement, parce que ce qu'elle avait à elle, en soignant son fils malade elle l'a tout utilisé et fini ». Et rapporte-moi ça de bonne heure parce que j'en ai besoin pour me frictionner avant d'aller à une séance au théâtre des dieux ${ }^{4}$.

5 Saisie de désespoir face à cette mission dont aucun mortel ne peut revenir vivant, Psyché veut se précipiter d'une tour, mais celle-ci s'anime et lui donne des conseils pour éviter les pièges mortels tendus par Vénus sur le périple des Enfers. La tour la rend attentive au piège de la boîte à remplir de ce "petit peu de beauté " qu'elle désigne, bien plus solennellement, comme un "trésor de beauté divine »: "Mais surtout, recommandation essentielle, fais bien attention à ça, ne pas essayer d'ouvrir la capsule que tu rapporteras, ni de regarder dedans, ni de savoir quoi que ce soit sur le trésor de beauté divine caché à l'intérieur ${ }^{5}$. " « Envahie par une curiosité invincible ${ }^{6}$ ", que Vénus avait bien prévue dans son plan perfide, Psyché ne peut résister et elle ouvre la boîte pour voir « le trésor de beauté divine ».

Giambattista Basile, qui connaît très bien le texte d'Apulée ${ }^{7}$, développe cet épisode d'une façon particulière qui n'échappera ni à Perrault ni à La Force. Dans «Le tre corone », la princesse Marchetta, enlevée par le vent comme Psyché, est déposée non pas dans le palais de son prétendant, mais directement dans la maison rustique d'une ogresse, dans laquelle il n'est pas difficile de reconnaître une reconfiguration grotesque de Vénus et de ses envies meurtrières transformées en cannibalisme. Lorsque l'ogresse 
découvre la présence de la jeune fille, qui s'est appliquée à la servir en cachette pour se rendre indispensable et pour éviter d'être dévorée par elle, elle lui adresse les paroles suivantes :

Tu as travaillé comme un maître et tu as évité de finir engloutie dans ma panse. Puisque tu as su si bien travailler et m'as donné tant de plaisir, je veux te considérer comme ma propre fille: voici les clefs de toute la maison, sois-en la maitresse archimaitresse. Je n'y mets qu'une restriction: ceci est la clef de la dernière chambre, ne l'ouvre sous aucun prétexte, car tu me ferais monter la moutarde au nez.

Perrault reprend de Basile cette extension de l'épisode du récit latin avec la transformation de la boite à ne pas ouvrir en chambre à ne pas ouvrir, ainsi que la scène des clés, tout en modifiant l'intrigue à son tour de façon significative ${ }^{9}$. Il prête le discours de l'ogresse napolitaine à un riche roturier s'adressant à sa jeune épouse et il transpose la scène dans une opulente demeure meublée dans le style de l'Ancien Régime :

Voila, luy dit-il, les clefs des deux grands garde-meubles, voilà celles de la vaisselle d'or \& d'argent qui ne sert pas tous les jours, voilà celles de mes coffres forts, où est mon or \& mon argent, celles des cassettes où sont mes pierreries, \& voilà le passepar-tout de tous les appartemens: pour cette petite clef-cy, c'est la clef du cabinet au bout de la grande gallerie de l'appartement bas: ouvrez tout, allez par tout, mais pour ce petit cabinet je vous deffend d'y entrer, \& je vous le deffens de telle sorte, que s'il vous arrive de l'ouvrir, il n'y a rien que vous ne deviez attendre de ma colère ${ }^{10}$.

Par un procédé ingénieux de redistribution des rôles, Perrault fusionne dans son personnage les traits de la perfide Vénus d'Apulée et ceux du portrait-robot du mari abject qu'elle souhaite à sa rivale. L'académicien ne manque pas de signaler le caractère intertextuel de cette fusion en dotant son personnage d'un attribut frappant, repéré dans le passage du texte latin qui suit immédiatement l'évocation d'un tel mari par Vénus. La déesse, avait précisé le narrateur d'Apulée, «s'en va vers la mer où elle est aussitôt entourée par les dieux marins qui s'empressent de la servir ${ }^{11} »$. Parmi eux se trouve « Portunus tout hérissé d'une barbe bleue ${ }^{12}$ ", Dieu romain des ports, des passes, mais aussi des portes que l'iconographie romaine représente avec une clé, symbole de son pouvoir divin sur les portes. Perrault dote son propre protagoniste de la barbe bleue et des clés du Dieu romain présenté comme le serviteur fidèle de Vénus ${ }^{13}$ et il lui prête ironiquement les gestes et les paroles d'une autorité divine, qui ne manquent pas d'intimider sa jeune épouse :

Estant arrivée à la porte du cabinet, elle s'y arresta quelque temps, songeant à la deffense que son Mari luy avoit faite, \& considerant qu'il pourroit luy arriver malheur d'avoir esté désobéissante; mais la tentation estoit si forte qu'elle ne put la surmonter: elle prit donc la petite clef, \& ouvrit en tremblant la porte du cabinet ${ }^{14}$.

9 La formulation «mais la tentation estoit si forte qu'elle ne put la surmonter » reprend l'idée de l'emprise de la curiosité invincible suggérée par le texte latin, mais le choix des termes "tentation » et " désobéissante » est aussi une allusion à la scène biblique de la tentation d'Ève. Cette connotation est d'autant plus ironique qu'elle attribue l'autorité absolue à un perfide roturier, meurtrier en série. L'autorité que le sombre personnage exerce sur sa femme se fonde en effet uniquement sur la fortune qui lui a permis d'obtenir les faveurs de la fille de noblesse désargentée. La Barbe bleue avait demandé à la mère, "dame de qualité », la main d'une de ses filles sachant que la nécessité de résoudre les problèmes d'une famille noble désargentée incombait 
inévitablement à une des deux. Dans l'Apologie des Femmes parue en 1694, au moment de concevoir les contes en prose, Perrault avait sévèrement critiqué cette politique maritale.

Le spectacle d'horreur qui s'offre à la jeune femme, quand elle ouvre le cabinet interdit et se trouve face aux cadavres des femmes assassinées par son mari, ne laisse aucun doute sur les dangers de ce type de mariage, ni encore sur la prétention d'un scélérat qui s'arroge un droit absolu sur la vie de ses femmes:

D'abord elle ne vit rien, parce que les fenestres estoient fermées; aprés quelques momens elle commença à voir que le plancher estoit tout couvert de sang caillé, \& que dans ce sang se miroient les corps de plusieurs femmes mortes, \& attachées le long des murs. (C'étoit toutes les femmes que la Barbe bleuë avoit épousées \& qu'il avoit égorgées l'une après l'autre) ${ }^{15}$.

11 Les lecteurs contemporains de Perrault, capables d'établir le lien intertextuel avec le célèbre conte ancien, se rendent compte d'une différence parlante: Psyché ouvre la boîte sans trop de scrupules pour se servir elle-même de la "beauté divine ", afin de plaire davantage à son amant. Elle n'y trouve "rien qu'un sommeil infernal, un vrai sommeil de Styx qui, [...] sitôt libéré du couvercle, répand sur tous ses membres une épaisse vapeur léthargique, et l'étend captive sur le chemin, à la place même où elle posait le pied ${ }^{16} »$. Le texte latin résume l'effet de cette magie par l'image suggestive du «cadavre endormi » (dormiens cadauer) dont Perrault s'inspire pour «La Belle au bois dormant ", premier conte programmatique du recueil ${ }^{17}$. À la différence notable de la princesse (qui doit attendre cent ans) et aussi à la différence de la jeune noble violemment agressée par le roturier meurtrier, Psyché est aussitôt amoureusement ranimée par Cupidon. Le Dieu de l'Amour décide de faire appel à son père omnipuissant Jupiter en lui demandant de protéger le jeune couple de la méchante Vénus. Ce que Jupiter fait sans tarder : il rend Psyché immortelle par le don d'ambroisie, l'accueille sur l'olympe et Vénus doit s'accommoder avec sa belle-fille ainsi promue au même rang qu'elle.

12 La femme de la Barbe bleue, sauvée in extremis par ses deux frères, n'a pas cette chance. Terrorisée par ce qu'elle a vu et rescapée de la tentative de meurtre de son mari, elle est loin des sphères célestes de sa sœur intertextuelle latine. Grâce à sa curiosité et à la transgression de l'interdiction autoritaire du scélérat, elle accède toutefois à un bonheur tout terrestre. Héritière des richesses de la Barbe bleue, elle peut sauver sa famille sans se sacrifier elle-même, acheter des charges pour ses frères, fournir une dot à sa sœur Anne et - situation enviable et inhabituelle dans le contexte de l'Ancien Régime - se remarier avec un « fort honnête homme » de son choix.

En reconfigurant ainsi l'intrigue du conte ancien, Perrault met en œuvre un procédé intertextuel très particulier, qui consiste à introduire des différences significatives par rapport au script intertextuel et lui permet de critiquer implicitement la réalité sociopolitique de son époque. Cette critique n'a certainement pas échappé aux lecteurs et aux lectrices qui lisaient ses textes avec le « degré de pénétration » recommandé dans l'épître dédicatoire à la lectrice emblématique du recueil, Élisabeth Charlotte d'Orléans, nièce de Louis XIV, elle-même concernée de près par ces problèmes ${ }^{18}$.

14 Le sombre réalisme des terreurs d'un mariage conclu pour le seul intérêt financier représenté dans «La Barbe bleue» se démarque également de façon frappante de la situation décrite dans l'intertexte napolitain, «Le tre corone ", auquel Perrault recourt en même temps qu'au texte latin. La princesse Marchetta, menacée par la colère de 
l'ogresse, ne s'embarrasse pas des scrupules qui hanteront la femme de la Barbe bleue. Elle suit simplement son impulsion spontanée : «Mais une fois que l'ogresse s'en fut allée, la jeune fille fut chatouillée par la curiosité, et elle voulut voir ce qu'il y avait dans cette chambre interdite ${ }^{19}$. $\gg$ Ce qu'elle voit forme un contraste frappant avec la scène macabre par laquelle Perrault la remplacera cinquante ans plus tard :

Elle ouvrit la porte et vit trois jeunes filles, toutes habillées d'or, qui semblaient dormir, assises sur des sièges impériaux. C'était les trois filles de la Magicienne auxquelles leur mère avait lancé un charme, car elle savait qu'elles couraient un grand danger à moins qu'une fille de roi ne vienne les réveiller; c'est pourquoi, elle les avait enfermées là-dedans pour les soustraire au péril dont les étoiles les menaçaient ${ }^{20}$.

Marchetta, dont l'ogresse ignore l'identité princière, s'avère être la "fille de roi » attendue pour réveiller les trois filles habillées d'or ${ }^{21}$, bien différentes des femmes égorgées suspendues aux murs du cabinet interdit à la femme de la Barbe bleue. Dans cette scène autrement vivante, elles demandent aussitôt à manger et la princesse n'hésite pas à leur préparer des œufs :

Marchetta entra dans la chambre et, au bruit de ses pas, les trois filles se réveillèrent. Aussitôt réveillées, elles demandèrent à manger. Alors Marchetta prit trois œufs, les fit cuire sous la cendre et les leur donna, et elles, ayant repris un peu leurs esprits, voulurent sortir de la pièce pour prendre l'air ${ }^{22}$.

La scène du retour de l'ogresse ressemble à celle du retour de la Barbe bleue par l'accès de colère spontanée de ces deux personnages, mais à la différence très significative de son successeur, l'ogresse s'adoucit très vite devant l'indignation de la princesse qui décide de partir :

Entre-temps, l'ogresse était revenue et elle se mit dans une telle colère qu'elle donna une claque magistrale à Marchetta. Celle-ci s'offensa et décida sur le champ de prendre congé de l'ogresse pour aller par le vaste monde, errant et se désespérant, à la poursuite de sa destinée. L'ogresse essaya de la calmer par de bonnes paroles, en lui disant que c'était pour plaisanter, qu'elle ne le ferait plus, mais Marchetta ne céda pas d'un pouce et l'ogresse fut contrainte de la laisser partir. Cependant elle lui offrit une bague $[. .].]^{23}$.

17 Il est tout aussi significatif que la sœur intertextuelle française de Marchetta, soumise au pouvoir absolu attribué par le contrat marital de l'Ancien Régime au riche roturier, ne soit pas capable d'un tel geste émancipatoire. Elle ne sera sauvée que grâce à l'aide de sa sœur et de ses deux frères que le hasard fait arriver juste à temps pour empêcher son exécution.

\section{De Vénus et la Barbe bleue à la méchante Reine des Fées}

18 Le premier des Contes des contes de La Force, dont le titre dit bien le lien intertextuel avec le recueil de Basile (resté longtemps inaperçu de la critique), répond intertextuellement en même temps aux récits de Perrault, Basile et Apulée en opérant à son tour des déplacements significatifs par rapport à leurs intrigues ${ }^{24}$. "Plus belle que Fée » raconte l'histoire d'une jeune princesse ainsi nommée, qui suscite, à l'instar de Psyché, par sa beauté et le choix de son nom, la colère d'une puissante "Reine des Fées ». Celle-ci reprend le rôle de Vénus dans cette nouvelle reconfiguration française de la célèbre fabella latine. Après avoir été incarnée par l'ogresse de Basile et par le 
perfide roturier de Perrault, la déesse romaine laisse désormais place à une fée malveillante dont La Force peint le portrait suivant :

La Reine des Fées n'étoit pas une de ces bonnes Fées, qui sont les protectrices de la vertu, \& qui ne plaisent qu’à bien faire. Après le cours de plusieurs siecles, elle étoit parvenuë à la Royauté par son grand sçavoir, \& par son artifice. Le nombre de ses ans l'avoit renduë fort petite, \& l'on l'appelloit plus que Nabote ${ }^{25}$.

La Reine des Fées s'en prend à Plus belle que Fée, nouvelle sœur intertextuelle de Psyché, en utilisant une ruse encore plus perfide que Vénus. Après s'être introduite comme servante "dans les bonnes grâces " de la Princesse, bien gardée par une Gouvernante dans un palais résistant à tout acte de magie, elle la fait sortir de l'enceinte du palais en feignant un malaise pour l'enlever de force :

[...] à peine la malheureuse fut-elle hors de cette fatale porte, que Nabote se releva, la saisit d'un bras puissant, \& faisant un cercle avec sa baguette, il se forma un broüillard épais \& noir, qui s'étant aussitôt dissipé, la terre s'ouvrit, il en sortit deux taupes, avec des ailes de feüilles de roses qui trainoient un char d'ébeine, \& se mettant dedans avec Plus belle que Fée, elle s'éleva en l'air, \& le fendit avec une vitesse incroyable, se perdant incontinent aux yeux des jeunes filles, qui par leurs pleurs \& leurs cris annoncerent bientôt dans tout le Château la perte qu'on venoit de faire ${ }^{26}$.

La Force recourt ici au même procédé intertextuel que Perrault: elle redistribue et fusionne les rôles du script latin en représentant la Reine des Fées en Pluton qui enlève Proserpine sur le modèle du rapt décrit dans les Métamorphoses d'Ovide ${ }^{27}$. Incité par Vénus, avide d'étendre son empire sur le Dieu des Enfers et sur la jeune fille qui veut rester vierge (filia virgo erit), l'enlèvement de Proserpine est, dans le récit d'Ovide, à la fois l'initiative de Vénus et de Pluton qu'elle fait frapper par une flèche de Cupidon ${ }^{28}$. La scène de l'enlèvement de la jeune fille par la Reine des Fées fusionne ainsi les actes de Vénus et de Pluton, comme Perrault avait auparavant fusionné Vénus et Portunus dans les gestes et attributs prêtés à la Barbe bleue. Le remplacement des chevaux noirs du char du Dieu des Enfers par les taupes aux ailes de feuilles de rose opère le transfert du monde mythologique au monde féerique sur un mode ironique, qui caractérise nombre de reconfigurations d'éléments mythologiques dans les contes français de la dernière décennie du XVII ${ }^{e}$ siècle. Proserpine joue un rôle important dans la fabella de Psyché. Elle y est représentée comme épouse du Dieu des Enfers, dans une phase de son existence ultérieure au rapt par Pluton, tel qu'il est évoqué dans le récit d'Ovide. En rappelant, par la description de l'enlèvement, l'ancien rôle de victime de Proserpine, le conte de La Force introduit dans l'intrigue de son conte de nouvelles situations et figurations qui entretiennent des relations significatives avec celles des récits d'Apulée, de Basile et de Perrault, tout en s'en distinguant.

Plus belle que Fée est secourue par un jeune homme qui apparaît dans une scène qui renvoie explicitement aux nombreuses représentations iconiques de Cupidon :

Toute la galerie fut dans un instant éclairée, \& elle vid à genoux devant elle un jeune garçon si beau \& si agreable, qu'à l'habillement prés elle le prit pour l'amour. Mais elle se souvint qu'on peignoit l'amour tout nud, \& ce beau garçon avoit un habit tout couvert de pierreries. [...]. Ce jeune garçon la considéroit toûjours à genoux, elle s'y voulut mettre aussi: qui êtes vous luy-dit-elle toute étonnée! êtesvous un Dieu, êtes vous l'amour? ${ }^{29}$

Dans un jeu intertextuel qui ne se dissimule pas, le conte de La Force reprend la clé de la Barbe bleue pour la donner à ce Cupidon tout habillé, bien plus pudique que son frère intertextuel antique. Il se présente comme Phraates, fils de la méchante Reine des Fées 
qui a repris le rôle de Vénus. Décidé à libérer la nouvelle Psyché de l'emprise de sa haineuse mère, il fait de la petite clé (recouverte d'or par les soins de La Force) un usage significativement différent de la Barbe bleue : il la donne à Plus belle que Fée non pas pour tenter sa curiosité de façon perfide, mais pour lui venir en aide dans sa situation difficile. La scène qu'elle découvre dans le cabinet ouvert par la petite clé d'or se distingue à son tour radicalement du spectacle des femmes égorgées du récit de Perrault :

[...] Plus Belle que Fée [...] repassa dans sa chambre, et trouvant le carré où était la serrure dont on lui avait parlé, elle l'ouvrit avec la clé d'or, et entra dans une chambre, dont la magnificence la surprit et la toucha, parce qu'elle y vit partout les soins de son amant. Il y avait [...] une table couverte de tout ce qui pouvait contenter la délicatesse du goût $[. . .]^{30}$.

Tout en rappelant l'accueil de Psyché dans le palais de son invisible prétendant, cette scène oppose à la vision sombre des dangers des mariages conclus par intérêt financier une image plus idéaliste et plus sentimentale de la relation amoureuse. C'est grâce au "véritable amour ", à la constance du sentiment amoureux et au respect mutuel que Plus belle que Fée en nouvelle Psyché et Phraates en Cupidon aimant parviendront ensemble à destituer la méchante Vénus-Reine des Fées et à restituer le règne de la bonne Reine des Fées, « protectrice de la vertu », qui l'avait précédée.

\section{De Vénus, la Barbe bleue et la Reine des Fées à la Vierge Marie}

"Marienkind" (Enfant de Marie), célèbre troisième des Kinder- und Hausmärchen, gesammelt durch die Brüder Grimm, se fonde sur un récit que Gretchen Wild avait raconté de vive voix aux Grimm en 1807, transcrit à la main par Wilhelm Grimm sur une des feuilles de l'ensemble envoyé en 1810 à Clemens Brentano ${ }^{31}$. La narration de la jeune femme s'ouvre sur l'évocation d'un pauvre bûcheron qui craint ne plus pouvoir nourrir sa fille de 3 ans. Au milieu de la forêt, il se trouve subitement face à " une belle femme », portant " une couronne pleine d'étoiles » (« eine Krone von lauter Sternen ») et une «robe bleu claire parsemée d'étoiles argentées» («ihr Kleid war himmelblau mit silbernen Sternen besät $\aleph^{32}$ ), qui se donne à connaître comme la Vierge Marie. Elle propose au bûcheron de prendre l'enfant avec elle, l'emmène au Ciel et lui donne des "vêtements dorés ", qui rappellent ceux des trois jeunes filles aperçues par Marchetta dans la chambre interdite. La Vierge Marie lui donne aussi, à l'âge de 14 ans, les fameuses clés que la Barbe bleue avait empruntées à l'ogresse de Basile et que La Force avait déjà pris soin de recouvrir d'or :

La Vierge Marie prit l'enfant avec elle dans le ciel. Elle y reçut des habits en or et les anges venaient jouer avec elle. Ainsi elle vécut longtemps en grande joie et magnificence jusqu'à sa quatorzième année. Alors, la Vierge Marie devant faire un long voyage alla vers l'enfant et dit: «Chère enfant, je dois faire un long voyage, voici les clés dorées, tu peux ouvrir toutes les portes du ciel et y entrer, sauf dans une seule, celle que cette petite clé-ci ouvre »; ensuite elle partit et laissa l'enfant seule $^{33}$. et la jeune fille selon le script intertextuel bien établi :

L'enfant prit les clés, ouvrit chaque jour une autre porte et se réjouit en voyant tous ces beaux appartements célestes. Enfin elle avait ouvert toutes les portes, il ne 
restait que celle qui était interdite: longtemps elle ne voulut pas [céder] mais

finalement elle ne put résister à sa curiosité ${ }^{34}$. $\mathrm{du}$ «trésor de beauté divine» que Psyché croit rapporter à la déesse Vénus. Il est toutefois frappant que la présence de la Sainte Trinité dans la chambre interdite par la Vierge Marie, en tant que présence exclusivement masculine constituée de l'union entre Dieu le Père, le Fils et le Saint Esprit, se substitue ici intertextuellement à trois chambres interdites exclusivement occupées par des femmes (dormantes, mortes ou en danger). Si nous comparons ces scènes en nous interrogeant sur les raisons pour lesquelles elles sont interdites à Marchetta et à l'épouse de la Barbe bleue, on peut se demander ce que la vision de la «splendeur indescriptible» de la Sainte Trinité découverte par l'enfant de Marie peut avoir de si compromettant et de punissable. Quel secret dangereux révèle cette vision, en quoi est-elle transgressive ? S'agit-il peut-être pour la Vierge Marie d'entourer de silence l'élucidation du dogme de sa virginité conservée grâce au Saint Esprit associé à Dieu le Père ? La difficulté de comprendre ce dogme et l'absence de toute explication de la part de la Vierge Marie, sa mère adoptive, pourrait-elle expliquer la réaction de défi que Gretchen Wild lui attribue en ces termes?

Peu après la vierge Marie revint de voyage, et après avoir récupéré les clés, elle demanda: «Est-ce que tu n'aurais pas ouvert la porte interdite? « Non», dit l'enfant. Marie lui posa alors sa main sur le cœur qui battait si violemment qu'elle vit bien qu'elle avait quand même transgressé l'interdit. Elle demanda encore une fois, mais l'enfant répondit à nouveau : « Non, ce n'était pas moi. $»^{37}$

A linsistance de la Vierge Marie, qui repose deux fois la même question, le récit de Wild oppose la réponse de l'enfant: «Non, ce n'était pas moi» («nein ich bin es nicht gewesen »). Cette réponse dit bien qu'elle ne se considère pas comme l'auteur d'un acte qu'elle n'a pas choisi d'exécuter, mais auquel elle semble avoir été contrainte par « l'irrésistible curiosité » émanant peut-être de la nécessité de comprendre une énigme inexplicable et inexpliquée par sa mère adoptive. La description de la stratégie et des moyens mis en œuvre par la Vierge Marie pour briser ce défi se greffe intertextuellement sur les actes et les paroles que Basile, Perrault et La Force avaient attribués à Vénus, à l'ogresse et au roturier meurtrier. La 
Vierge Marie prive sa fille adoptive de la possibilité de s'exprimer en la rendant muette et en l'expulsant de sa demeure céleste. La fille muette se retrouve dans une contrée sauvage, réduite à se nourrir de baies et à dormir sous un arbre. Un prince la trouve, l'emmène dans son château et l'épouse. La Vierge Marie ne revient vers elle qu'au moment où elle accouche de son premier enfant : elle lui enlève son enfant, parce que la jeune femme n'admet toujours pas avoir été l'auteure de l'acte incriminé. La Vierge Marie fait de même avec les deux enfants suivants, causant ainsi la condamnation au bûcher de la jeune femme soupçonnée d'avoir tué ses enfants. Quand les flammes l'atteignent, une pensée vient à la reine malheureuse que Gretchen Wild formule dans une phrase qui reste lacunaire dans la transcription manuscrite : " 0 wie gern wollte ich jetzt alles gestehn [, könnt ich mein/e Kinder] ${ }^{38} . »:$ "Oh! comme j'admettrais volontiers tout [si je pouvais voir/avoir mes enfants].» On peut comprendre que c'est le désir de voir ses enfants au moment de mourir sur le bûcher qui lui fait avouer un acte dont elle pense ne pas avoir été l'auteure. La narration de Gretchen Wild semble hésitante à cet endroit: la phrase laisse en effet entendre que l'aveu serait ici stratégique face au comportement de la Vierge Marie toujours ressenti comme injuste.

Notons que dans la version imprimée de 1812, Wilhelm Grimm accentue la gravité et la teneur religieuse de cette transgression dans la narration faite de vive voix par Gretchen Wild. Il modifie le syntagme : «longtemps elle résista à sa curiosité » (« lange widerstand es seiner Neugier») en " longtemps elle fut pieuse et eut peur» ("lange war es fromm und scheute sich»). "Être curieux» devient alors synonyme de "ne pas être pieux »: la connotation biblique teintée d'ironie dans la représentation de la Barbe bleue par Perrault est ici reprise au premier degré par Grimm, qui l'ajoute au récit de Gretchen Wild dont cette connotation était absente. Cette modification s'inscrit dans une série d'autres apportées au texte de 1810 par Wilhelm Grimm, dès 1812, manifestement destinées à aggraver la faute de l'enfant afin de légitimer la dureté disproportionnée dont la Vierge Marie fait preuve à l'égard de sa fille adoptive. À cette fin, Grimm exploite davantage le script intertextuel bien connu de "La Barbe bleue » dans sa réécriture de 1812 du récit de Gretchen Wild ${ }^{39}$. L'enfant de Marie ne s'y contente pas d'ouvrir la porte et de la refermer, mais elle touche du doigt la lumière dorée entourant l'apparition de la Sainte Trinité. Son doigt se teinte d'or et devient ainsi la preuve ineffaçable de son "péché ", preuve que la narration de Gretchen Wild avait justement omis de fournir. Par cet ajout, Wilhelm Grimm présente la fille du bûcheron comme une menteuse obstinée et désormais repentie de manière à effacer toute ambiguïté au sujet du comportement de la Vierge Marie, à la différence de sa narratrice d'origine huguenote. Cette différence traduit peut-être aussi un problème plus général de compréhension par les huguenots du statut de la Vierge Marie dans les dogmes catholiques.

\section{Pour conclure}

Notre parcours intertextuel et interculturel nous a menés de Vénus à la Vierge Marie, en passant par une ogresse, un tueur en série et une méchante Reine des Fées. Qui l'emporte dans ce palmarès de la perfidie et de l'ambiguïté déployée par ces personnages issus d'imaginaires et de mythologies différents? Serait-ce la Vierge Marie? Une telle conclusion relèverait de l'ontologisation de ces personnages que les résultats de l'analyse textuelle et intertextuelle mettent précisément en cause. La 
comparaison différentielle précise des textes montre que les personnages, tels qu'ils apparaissent dans les contes, sont des fabrications textuelles et intertextuelles, émanant d'un bricolage ingénieux de passages de textes, d'épisodes, de rôles et de discours redistribués, de phrases et métaphores frappantes que les recueils et ouvrages cités empruntent les uns aux autres en les reconfigurant de façon inventive. Ces recueils et textes ont circulé dans toute l'Europe et au-delà par le biais du colportage, des éditions piratées, des anthologies et de re-narrations orales, et ils sont entrés dans l'interdiscours et la mémoire collective, ce qui n'a fait qu'augmenter leur caractère de palimpseste et leur potentiel suggestif. Dans ce jeu intertextuel, interdiscursif et interculturel incessant se créent de nouvelles histoires et de nouvelles figures à partir de celles qui existent déjà : elles se ressemblent de façon surprenante, mais elles diffèrent de façon significative par les effets de sens dont elles sont investies. Ces effets de sens ne leur sont pas intrinsèques ; ils relèvent, comme nous l'avons vu, des projets esthétiques, génériques et des paradigmes religieux et socio-culturels différents et différenciés de leurs auteurs, narrateurs, éditeurs et autres ré-énonciateurs, et des époques, langues et cultures dont ils émanent.

\section{NOTES}

1. Pour la définition du concept de dialogue intertextuel et de son importance pour l'histoire du genre conte, je me permets de renvoyer à mes études, «Expérimentation générique et dialogisme intertextuel : Perrault, La Fontaine, Apulée, Straparola, Basile ", Féeries, n 8, "Le merveilleux français à travers les siècles, les langues, les continents", dossier coordonné par J. Mainil, Grenoble, Ellug, 2011, p. 45-69, ainsi que "Le dialogisme intertextuel des contes des Grimm », Féeries, $\mathrm{n}^{\circ} 9$, «Le dialogisme intertextuel des contes des Grimm», dossier coordonné par U. Heidmann, Grenoble, Ellug, 2012, p. 9-28.

2. Apulée, Les Métamorphoses ou l'Âne d'or, texte établi par D. S. Robertson, présenté et traduit par O. Sers, Paris, Les Belles Lettres, 2007, p. 1-2 et 242-243.

3. Voir à ce sujet V. Gély, "Les renaissances de Psyché ", dans Psyché à la Renaissance, textes réunis et édités par M. Bélimé-Droguet, V.Gély, L. Mailho-Daboussi et P. Vendrix, Turnhout, Brepols, coll. «Études renaissantes », 2013, p. 7-19.

4. Apulée, ouvr. cité, ici p. 230-233 : «Sed adhuc istud, mea pupula, ministrare debebis. Sume istam pyxidem, et dedit; "protinus usque ad inferos et ipsius Orci ferales penates te dirige. Tunc conferans pyxidem Proserpinau: "Petit de te Venus" dicito "modicum de tu mittas ei formonsitate uel ad unam saltem dieculam sufficiens. Nam quod habuit, dum filium curat aegrotum, consumpsit atque contriut omne". Sed haud immaturius redito, quia me necesse est indidem delitam theatrum deorum frequentare. »

5. "Sed inter omnia hoc obseruandum praecipue tibi censeo, ne uelis aperire uel inspicere illam quam feres pyxidem uel omnino diuinae formonsitatis abditum curiosius thensaurum.» (Apulée, ouvr. cité, p. 236-237).

6. «[...] mentem capitur temeraria curiositate [...]» (Apulée, ouvr. cité, p. 236-237).

7. Dans l'étude de Féeries $n^{\circ} 8$ citée plus haut, je rends attentif au rapport intertextuel du recueil de Basile aux Métamorphoses d'Apulée.

8. G. Basile, Le Conte des contes ou le divertissement des petits enfants, traduit du napolitain par F. Decroisette, Strasbourg, Circé, 1995, p. 349. « Ma, pocca hai saputo fare tanto e m'hai dato gusto, io 
te voglio tenere chiù che figlia: perzò eccote le chiave de le cammare e singhe domene e domenanzio. Sulo me reservo na cosa: che non vuoglie aprire ' $n$ cunto nesciuno l'utema cammara, dove va bona sta chiave, che me farisse saglire buono la mostarda a lo naso. » (G. Basile, Lo Cunto de li cunti, A cura di Michele Rak, con testo napoletano e traduzione a fronte, Milan, Garzanti, [1998] 2006, p. 760-761). Comme pour le texte latin, il importe de mener l'analyse à partir du texte de Basile dans la langue d'origine. Selon Suzanne Magnanini, les auteurs français ont eu accès au Cunto de li cunti, voir «Telling Tales out of School: The Fairy Tale and Italian Academies », The Romanic Review, vol. 3-4, 2008, p. 257-270.

9. Si la critique a longtemps supposé que l'académicien était incapable de lire le napolitain, les analyses intertextuelles que j'ai menées dans les études référenciées dans cet article infirment ce préjugé, tant ses réponses intertextuelles sont précises et se réfèrent à la lettre du texte.

10. C. Perrault, Histoires ou contes du temps passé. Avec des Moralitez, Paris, Barbin, 1697, p. 62-63.

11. "Set statim, quasi pridem praeceperit, non moratur marinum obsequium " (Apulée, ouvr. cité, p. 160-161).

12. «Portunus caereulis barbis hispidus » (Apulée, ouvr. cité, p. 160-161).

13. J'ai montré ailleurs que cette sorte de bricolage intertextuel est un moyen très ironique pour opposer à Nicolas Boileau, défenseur d'une imitation jugée servile des Anciens au service de l'idéologie du pouvoir absolu de Louis XIV, une tout autre façon, résolument moderne, de se référer aux textes anciens (U. Heidmann, "Intertextualité et dialogicité des contes", dans U. Heidmann et J.-M. Adam, Textualité et intertextualité des contes. Perrault, Apulée, La Fontaine, Lhéritier..., Paris, Classiques Garnier, 2010, p. 142-149).

14. C. Perrault, ouvr. cité, p. 66-67.

15. Ibid., p. 69.

16. «[...] qui statim coperculo reuelatus inuadit eam crassaque soporis nebula cunctis eius membris perfunditur et in ipso uestigio ipsaque semita conlapsa<m> possidet; Et iacebat immobilis et nihil aliud quam dormiens cadauer » (Apulée, ouvr. cité, p. 236-237).

17. Voir U. Heidmann, «Expérimentation générique et dialogisme intertextuel », art. cité, p. $52-62$.

18. J'analyse cette problématique plus en profondeur dans « Histoire ou conte du temps passé et critique du temps présent: La Belle au bois dormant dédiée à la nièce de Louis XIV », dans M. Hersant et R. Jourmand-Baudry (éd.), Conte et histoire, 1690-1800, Paris, Classiques Garnier, 2017, p. 241-267.

19. "Ma partita l'orca, se sentie tillicare grannamente la curiosità de verdere che 'nce fosse drinto a chella cammara proibita [...]. » (G. Basile, Lo Cunto de li cunti, ouvr. cité, p. 760). La traduction de ce passage se trouve dans G. Basile, Le Conte des contes, ouvr. cité, p. 350.

20. Ibid., p. 350. "[...] ed, apertola, 'nce trovaie tre figliole, vestute tutte d'oro, sedute a tre segge a l'imperiale, che paravano che dormessero. Erano cheste tutte figlie de la fata 'ncantate da la matre perché sapeva c'avevano da passare no gran pericolo si no le veneva a scetare na figlia de re e perzò l'aveva 'chiuse là drinto, pe levarele da lo riseco che le ammenacciavano le stelle. » (G. Basile, Lo Cunto de li cunti, ouvr. cité, p. 760-763).

21. Perrault (pensant peut-être à cette scène des "Tre corone ») invente une scène dans «La Belle au bois dormant ", dans laquelle l'Arrêt des Fées annonce « un fils de roi » pour réveiller la Belle après cent ans, comme je l'expose dans « Histoire ou conte du temps passé et critique du temps présent : La Belle au bois dormant dédiée à la nièce de Louis XIV », art. cité, note 20.

22. G. Basile, Le Conte des contes, ouvr. cité, p. 350. « Ora, trasuto là dintro Marchetta, a lo remmore che fece co li piede chelle se resentettero, comme se scetassero, e le cercaro da magnare. Ed essa pigliato subito tre ova ped uno e fattole cocere sotto le cennere, ce le dette. " (G. Basile, Lo Cunto de li cunti, ouvr. cité, p. 762-763).

23. G. Basile, Le Conte des contes, ouvr. cité, p. 350. «[...] tra lo quale tiempo arrivato l'orca ebbe tanto degusto che schiaffaie no boffettone a Marchetta, la quale se ne pigliaie, tanto affrunto c'a la medesema ora 
cercaie lecenzia all'orca de se partire pe ire sperta e demerta pe lo munno, cercanno la sciorte soia./ Pe quanto cercaie l'orca d'accorderela de belle parole, decenno c'aveva abborlato e ca no lo voleva fare chiù, non fu possibile a levarela de pede, tanto fu costretta a lasciarla partire, dannole n'aniello. » (G. Basile, Lo Cunto de li cunti, ouvr. cité, p. 762-763).

24. «Plus belle que Fée » répond intertextuellement encore, comme les Histoires ou contes du temps passé de Perrault, aux Amours de Psiché et de Cupidon de La Fontaine (1669), autre strate intertextuelle importante, que l'espace restreint de cet article ne me permet pas de déployer.

25. [La Force, Charlotte-Rose de Caumont], Les Contes des contes. Par Mademoiselle de ***, À Paris, Chez S. Bernard, 1698, p. 5.

26. Ibid., p. 11-12.

27. L'ouvrage d'Ovide était très connu à la cour de Louis XIV qui s'entourait de peintures et de tableaux représentant des scènes et figures mythologiques dans lesquelles le Roi se faisait représenter lui-même, ainsi que ses maîtresses et les membres de la famille royale.

28. Voir Métamorphoses, liv. V, v. 375 : «Vénus à Cupidon : Ne vois-tu pas que Pallas et Diane la chasseresse/ Ont refusé ma loi ? La fille de Cérès même, si nous le supportons,/ Restera vierge (Cereris quoque filia virgo, Si patiemur, erit; nam spes affectat easdem) ", v. 375 et suiv., Arles, Actes Sud, coll. "Thesaurus ", p. 212. Cet épisode des Métamorphoses d'Ovide était sans doute reconnu comme intertexte par les lecteurs et lectrices cultivés de La Force, habitués par La Fontaine à ce type de jeux intertextuels.

29. La Force, ouvr. cité, p. 22.

30. Ibid., p. 96.

31. Cet ensemble de textes manuscrits de 1810 a été édité par Heinz Rölleke dans Die Älteste Märchensammlung der Brüder Grimm. Synopse der handschriftlichen Urfassung von 1810 und der Erstdrucke von 1812, Cologny-Genève, Fondation Martin Bodmer, 1975. Le récit de Gretchen Wild transcrit par la main de Wilhelm Grimm, par la suite référencé comme " Marienkind 1810 », se trouve aux pages 196, 198 et 200 de cette édition. Il y est mis en regard de la version significativement modifiée par Grimm pour la première édition imprimée des Kinder- und Hausmärchen de 1812, référenciée ici comme «Marienkind 1812 ». Ce deuxième texte se trouve aux pages 197, 199 et 201 de l'édition de Rölleke.

La transcription porte encore des traces évidentes des intertextes français. Les corrections et modifications, apportées à ce texte dans l'édition de 1812 en vue de l'édition de 1819, illustrent bien la tentative de Grimm de « christianiser » le récit et de recouvrir par ce biais sa trop grande proximité avec les intertextes français. Notre texte de référence manuscrit de la main de Wilhelm Grimm (avec deux annotations dans la marge de la main de Jacob Grimm) a été édité par Rölleke en 1975, Die Äelteste Märchensammlung, ouvr. cité, en face du texte publié en 1812, p. 196-201. Au fil des éditions successives, d'autres modifications ont été apportées qui renforcent les orientations prises en 1812.

32. Die Älteste Märchensammlung, ouvr. cité, « Marienkind 1810 », p. 196. Cette description rappelle non seulement les images pieuses de la Vierge Marie, mais aussi celle de la robe couleur ciel de «Peau d'Asne » de Perrault, à mon avis intertextuellement « greffée » sur l'évocation de la déesse Isis dans les Métamorphoses d'Apulée.

33. "Die Jungfrau Maria nahm das Kind mit sich in den Himmel. Dort erhielt es goldene Kleider und die Engel kamen und spielten mit ihm. So lebte es lang in großer Freude und Herrlichkeit bis in sein vierzehntes Jahr. Da mußte die Jungfrau Maria eine weite Reise thun, sie ging zu dem Kind und sprach: "liebes Kind ich muss eine weite Reise thun, hier hast $d u$ die goldenen Schlüßel, du darfst alle Thüren des Himmels aufschließen und hinein gehen, [aber] nur in eine einzige nicht, die dieser kleine Schlüßel aufschließt", darauf ging sie weg und lies das Kind allein. » (Ibid.).

34. "Dieses nahm die Schlüßel, öffnete jeden Tag eine andere Thür und freute sich wie es die schönen himmlischen Wohnungen all erblickte. Endlich hatte es alle Thüren aufgeschloßen, nur die verbotene war 
noch übrig: lange wollte es nicht endlich aber konnte es seiner Neugierde nicht widerstehen. » (Ibid., p. 196 et 198 , je traduis).

35. Ibid.

36. Les Contes des contes de La Force occupent le volume 6 du Cabinet des Fées de 1785 où Gretchen Wild, d'origine huguenote et parlant et lisant parfaitement le français, avait pu les lire, comme les Grimm eux-mêmes d'ailleurs.

37. «Bald darauf kam die Jungfrau Marie von der Reise zurück, und nachdem sie die Schlüßel wieder empfangen fragte sie: hast du auch nicht die verbotene Thür aufgeschloßen? Nein sagte das Kind. Da legte Maria die Hand auf sein Herz das schlug gewaltig, und sie sah wohl dass es doch das Gebot übertreten hatte. Sie fragte noch einmal, aber das Kind antwortete wieder: nein, ich bin es nicht gewesen. " (Die Äelteste Märchensammlung, ouvr. cité, « Marienkind $1810 »$, p. 198, je traduis).

38. « O wie gern wollte ich jetzt alles gestehn [, könnt ich mein/e Kinder]. » (Ibid., p. 200, je traduis).

39. Pour sa reconfiguration de la célèbre scène de "La Barbe bleue ", Grimm s'inspire de toute évidence aussi d'un récit intitulé «Ottilie », dû à Benedikte Naubert, inséré dans son recueil Neue Volksmärchen der Deutschen (Leipzig, 1789), qu'il mentionne dans une note. Faute de place, cette autre strate intertextuelle ne peut être déployée ici.

\section{RÉSUMÉS}

Le parcours analytique proposé ici mène de la déesse romaine Vénus, telle qu'elle est représentée dans le célèbre conte ancien de Psyché et Cupidon inséré dans les Métamorphoses (L'Âne d'or) d'Apulée, à l'ogresse qui prend sa place dans Le tre corone de Giambattista Basile. Des textes latin et napolitain, l'enquête conduit à $L a$ Barbe bleue de Charles Perrault, dont le protagoniste hérite à la fois du comportement perfide de la Vénus d'Apulée et des paroles de l'ogresse napolitaine. Dans Plus belle que Fée de Charlotte-Rose de Caumont de La Force, une méchante Reine des Fées relaye à son tour Vénus, l'ogresse et la Barbe bleue. Enfin, dans Marienkind (Enfant de Marie), troisième des Kinder- und Hausmärchen narré aux frères Grimm par Gretchen Wild, jeune femme d'origine huguenote, c'est la Vierge Marie qui reprend les paroles et les actes successivement attribués à Vénus, à l'ogresse, à la Barbe bleue et à la méchante Reine des Fées dans les récits d'Apulée, Basile, Perrault et La Force.

L'analyse comparative précise menée à partir de leurs langues et éditions d'origine montre que ces personnages sont des fabrications textuelles et intertextuelles émanant d'un bricolage ingénieux d'épisodes, de rôles et de discours redistribués, de phrases et de métaphores frappantes que leurs auteurs empruntent les uns aux autres en les reconfigurant de façon inventive. L'étude donne ainsi à voir le travail complexe de différenciation intertextuelle et interculturelle accompli d'un recueil et d'un texte à l'autre. Ces recueils et textes ont circulé dans toute l'Europe (et au-delà) par le biais du colportage, des éditions piratées, des anthologies et de re-narrations orales. Ils sont ainsi entrés dans l'interdiscours et dans la mémoire collective, ce qui n'a fait qu'augmenter leur caractère de palimpseste et leur potentiel suggestif.

The investigation proposed in this essay starts with the analysis of the Roman goddess Venus as she is represented in the famous ancient tale of Psyche and Cupid in the Metamorphoses (The Golden Ass) of Apuleius and then transformed into an ogress in the narrative Le tre corone by Giambattista Basile. From the Latin and Neapolitan texts, it leads to the French La Barbe bleue by Charles Perrault, whose protagonist inherits both the treacherous behaviour of Apuleius' Venus 
and the words of the Neapolitan ogress. In Plus belle que fée by Charlotte-Rose de Caumont de La Force, it is the turn of an evil Queen of the Fairies to re-enact Venus, the ogress and Bluebeard. Finally, in Marienkind (Mary's child), the third of the Kinder- und Hausmärchen related to the Grimm brothers by Gretchen Wild, it is the Virgin Mary that endorses the words and actions successively attributed to Venus, the ogress, Bluebeard and the evil Queen of the Fairies in the tales of Apuleius, Basile, Perrault and La Force.

The precise comparative analysis of the narratives as presented in their original languages and editions shows that the famous figures are ingenious textual and intertextual constructs that originate from an inventive assembly of episodes, of redistributed roles and discourses, of striking phrases and metaphors that their authors borrow from each other through creative reconfiguration. This study thus highlights the creative intertextual and intercultural differentiation at work in this process. These texts travelled all over Europe (and beyond) through peddling, pirated editions, anthologies, and oral retellings. They have thus entered collective memory while increasing their potential of suggestion.

\section{INDEX}

Mots-clés : Amours de Psiché et de Cupidon (La Fontaine), Apologie des Femmes (Perrault), Kinder- und Hausmärchen, gesammelt durch die Brüder Grimm, Le tre corone (Giambattista Basile), Lo cunto de li cunti (Giambattista Basile), Métamorphoses ou L'Âne d'or (Apulée), Plus belle que Fée (La Force), La Belle au bois dormant (Perrault), Contes des contes (La Force), Die Älteste Märchensammlung der Brüder Grimm, Apulée, Basile, Brentano, Wilhelm Grimm, La Fontaine, La Force, Perrault, Gretchen Wild, Barbe bleue, Cupidon, Jupiter, Marchetta, Marienkind, Proserpine, Ogresse, Plus belle que Fée, Portunus, Psyché, Vénus, Vierge Marie

\section{AUTEUR}

\section{UTE HEIDMANN}

Université de Lausanne, Littératures comparées (CLE) 